\title{
A novel mutation in CELSR1 is associated with hereditary lymphedema
}

\author{
Vascular Cell 8:5 । DOI: 10.1186/s13221-016-0035-5 I C Li et al.; licensee Publiverse Online S.R.L. 2016 \\ Received: 8 Oct 2015 | Accepted: 29 Oct 2016 | Published: 5 Oct 2016 \\ Gonzalez-Garay M. L., Aldrich M. B., Rasmussen J. C., Guilliod R., Lapinski P. E., King P. D., \\ Sevick-Muraca E.M. @ \\ + Contributed equally ${ }^{@}$ Corresponding author
}

\begin{abstract}
Background

Biological evidence reported in the literature supports the role of CELSR1 as being essential for valvular function in murine lymphatics. Yet thus far, there have been no variants in CELSRI associated with lymphatic dysfunction in humans.

\section{Case Presentation}

In this report, a rare early inactivating mutation in CELSRI is found to be causal for non-syndromic, lower extremity lymphedema in a family across three generations. Near-infrared fluorescence lymphatic imaging shows that instead of being propelled within the lumen of well-defined lymphatic vessels, lymph moved in regions of both legs in an unusual fashion and within sheet-like structures.
\end{abstract}

\section{Conclusion}

CELSRI may be responsible for primary, non-syndromic lymphedema in humans.

\section{Keywords}

Primary lymphedema - Whole exome sequencing - Near-infrared fluorescence imaging CELSR1 - Planar polarity

\section{Background}

Lymphedema (LE) is a condition caused by a defective lymphatic system where an excess of fluid accumulates and generates chronic swelling of tissues. Primary LE is a rare genetic disorder that can develop at birth (congenital), at puberty (praecox), or after 35 years of age (tarda) [ 1]. FLT4 was the first pathogenic gene identified in primary LE [ 2], but to date, there is only a handful of genes (FOXC2, CCBE1, GCJ2, SOX18, GATA2, PTPN14) associated with hereditary LE, with the majority associated with patients in which LE is part of a genetic syndrome [ 3]. Mendola et al. recently argued that the genetic cause(s) of the majority of families harboring hereditary LE has not yet been identified. In their group of 78 index patients with inherited LE who were screened for mutations in the top seven LE genes, they found explanations for only $36 \%$ of the cases [4].

In this report, we present the unusual lymphatic phenotype obtained by near-infrared fluorescence lymphatic imaging (NIRFLI) of a woman diagnosed non-syndromic, lower extremity LE and compare it with that of her asymptomatic mother. From clinical diagnoses and whole exome sequencing (WES) of family members across three generations, we found no gene variants known to be responsible for LE, but instead found a rare, inactivating mutation in CELSRI that could be causative for the inherited condition and the lymphatic phenotype imaged in the index case.

\section{Case Presentation}

\section{Family case report}

A 62 yo female previously diagnosed with primary, lower extremity LE (bilateral, Stage II by International Society of Lymphology staging system) and her 84 yo asymptomatic mother enrolled in an ongoing, institutional review board (IRB) 
and FDA-approved (IND\# 102,827) clinical study of lymphatic disorders (Clinical Trials No. NCT00833599: "Imaging lymphatic function in normal subjects and in persons with lymphatic disorders" www.clinicaltrials.gov). The index case self-reported onset of lower extremity swelling at age 10, with diagnosis of LE at age 39 at which time she began compression treatment. In addition, she was also previously diagnosed with type 2 diabetes, high cholesterol, and high blood pressure. Following consent, a clinical examination was first performed followed by near-infrared fluorescence lymphatic imaging (NIRFLI) [ 5] to assess lymphatic anatomy and function in the legs. Blood was collected for WES of DNA.Upon questioning, we further found a family history of lower extremity LE and traveled to the family's hometown to consent, conduct standard clinical examinations (including measurements of leg volumes and assessment of Stemmer's sign), and to collect blood from family members spanning three generations. We did not perform NIRFLI on these family members. Other than stage I and II bilateral lower extremity, non-syndromic LE, we found no remarkable evidence of disease among the family members.

\section{Imaging studies}

For lymphatic imaging of the daughter and mother, doses of $25 \mu \mathrm{g}$ of indocyanine green (ICG) in $0.1 \mathrm{cc}$ saline were administered bilaterally in the dorsum of the feet, medial ankles, the medial and lateral calves, and the thighs for a total dose of $300 \mu \mathrm{g}$. Images were acquired with exposure times of $200 \mathrm{~ms}$ and sequential images were compiled to assess for the presence of active lymphatic propulsion/movement of ICG-laden lymph from the injection sites proximally towards the inguinal lymph node basins. As shown by NIRFLI in Fig. 1b, the affected daughter had extensive areas of dermal backflow in her lower legs, as commonly observed in LE subjects, but possessed a unique, uncommon flow pattern of lymph movement that appeared in "sheets" rather than within distinct vessel lumens (Additional file 1: Video 1). While the proband's mother primarily had well-defined and linear lymphatics (Fig. 1b) as typically seen in healthy subjects [ 6, 7], she nonetheless presented with smaller areas of dermal backflow and tortuous lymphatics as shown in the insets of Fig. 1b. Active lymphatic propulsion of ICG-laden lymph was observed in the well-defined lymphatic vessels in the mother (Additional file 2: Video 2), in contrast to the limited lymphatic movement observed in the daughter.

Fig. 1
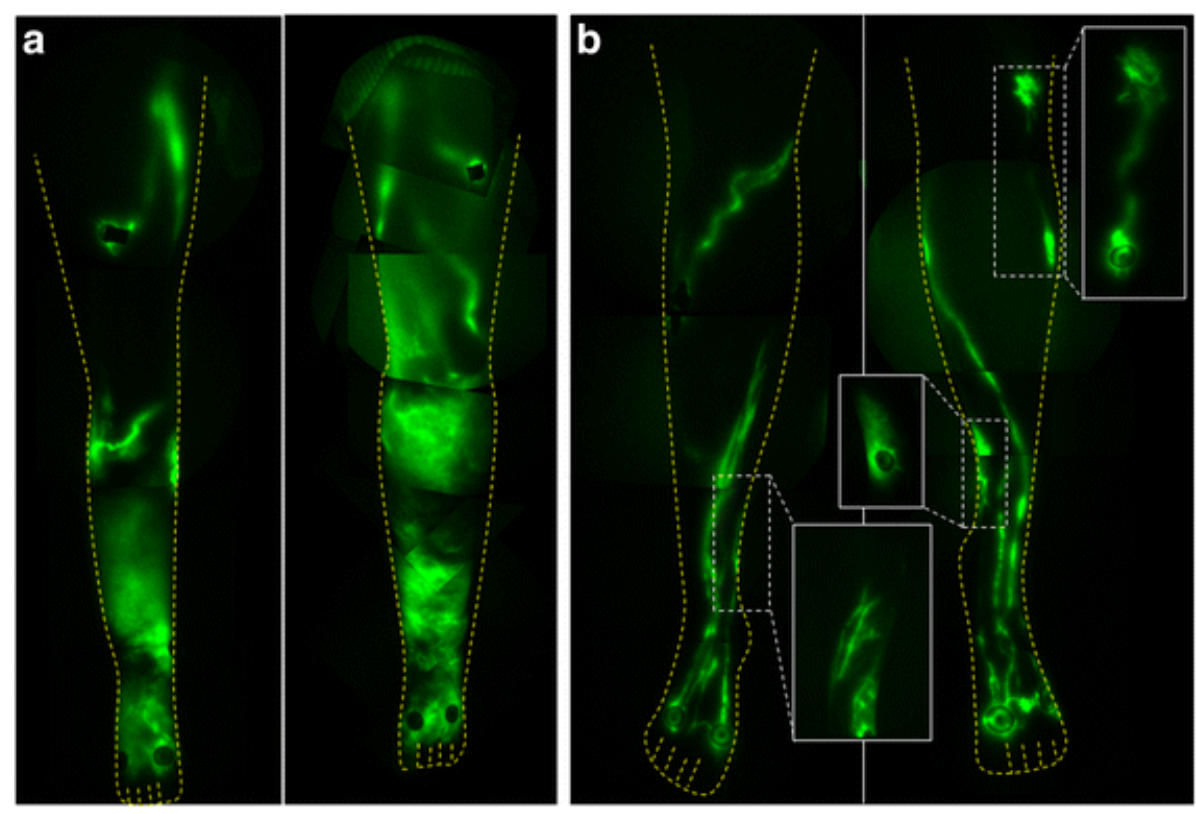

Montages of NIRFLI of the lower extremities of ( a) the proband and ( b) her mother. While the mother had welldefined lymphatics, she also had areas with dermal backflow as shown in the inset images of (b)

\section{Family evaluation}

In addition to the proband and her mother, DNA was collected from 9 family members (subjects 146-154) including 5 members diagnosed with LE upon clinical examination. The pedigree chart in Fig. 2 shows that the diagnosed disorder segregates in an autosomal dominant manner. The affected members exhibited grade II bilateral leg lymphedema, with the exception of subject 154, with grade I bilateral leg lymphedema. DNA was extracted from blood samples using Paxgene Blood DNA Kit (PreAnalytix, Switzerland) according to the vendor's instructions. $2 \mu \mathrm{g}$ of genomic DNA was submitted to Axeq Technologies for human exome capture sequencing using SureSelect Human All Exon V5 and V5 + UTRs (Agilent Technology, Santa Clara, CA). 


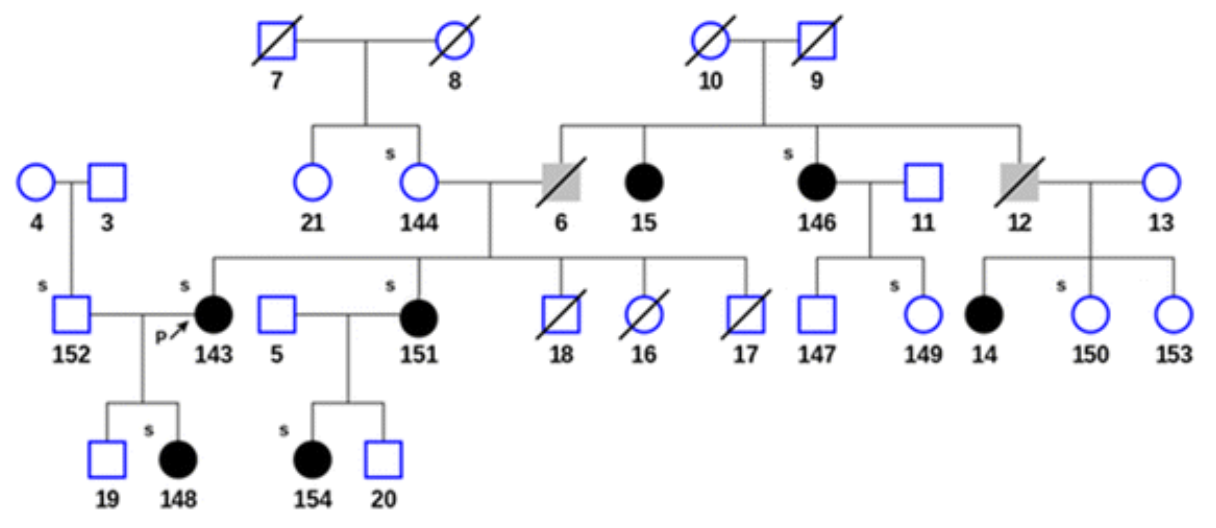

Pedigree of the family showing members diagnosed with lymphedema (filled black), unknown phenotype (filled gray) and unaffected (open) subjects. Arrow indicates the proband. The small 's' at the left top corner of the subjects indicates when the subject's DNA was sequenced. A diagonal line indicates that the patient is deceased

\section{Genetic sequencing analyses}

Genetic sequencing analysis was conducted as follows. Each pair of fastq files was aligned to human genome (hg19) using Novoalign (Novocraft Technologies; www.novocraft.com), keeping parameters at the default settings, as recommended by Novocraft Technologies. SAMtools ( http://samtools.sourceforge.net) was used to sort the SAM files, create BAM files, and generate their index files. Picard (SourceForge; http://broadinstitute.github.io/picard) was used to remove all of the PCR duplicates from the BAM files. For local realignments, base quality recalibration, and variant calling, we used Genome Analysis Toolkit (GATK) Version 3.1-1 [ 8]. Finally, for variant annotation, we used SnpEff ( http://snpeff.sourceforge.net/) [ 9], variant tools and ANNOVAR [ 10] using multiple databases from UCSC Genome bioinformatics. Functional effects for each non-synonymous coding variant was evaluated using three different functional prediction algorithms: (1) Polyphen 2.0 Prediction of functional effects of human nsSNPs ( http://genetics.bwh.harvard.edu/pph2) [ 11], (2) SIFT [ 12] and (3) ( www.mutationtaster.org) [ 13] using the dbNSFP database [14]. Filtration of common polymorphisms was accomplished using frequencies from the NHLBI Exome sequencing project (ESP) ( http://evs.gs.washington.edu/EVS) [ 15] and the 1,000 Genomes Project ( http://www.1000genomes.org/data) [ 16].

WES results showed that the proband did not have variants in any of the known genes previously associated with lymphatic dysfunction. To maximize the power of the segregation analysis, we selected family members diagnosed with LE from each generation for a total of 5 affected cases. Since we did not have controls of the same ethnicity, we selected 4 unaffected family members to reduce the background and increase the statistical power of the analysis. The raw results from the sequencing passed our standard quality control, and the variant files (vcf) reported a consistent number of variants. After selecting non-synonymous coding variants with low frequency (MAF $<0.1)$, we performed co-segregation analysis using a dominant model of inheritance. Our analysis generated six potential candidate variants, five of them were eliminated after a quick manual inspection with the Integrative genomics viewer [17]. We identified a nonsense coding variant that generated a prematurely terminated protein CELSR1 (p.W1957X, Hg19chr22:g.46,790,132C > T;

NC_000022.10:g.467901432C>T; NM_014246.1:c.5871G > A). The variant was private to the family and has not been reported in any of the following databases: 1000 Genomes [ 16], ESP6500 [ 15],UK10K, TCGA germline [ 18], Scripps Wellderly [ 19], ExAC [ 20], dbNSFP [ 14], ClinVar [ 21], OMIM [ 22], COSMIC [ 23], and Reference Variant Store (RVS), [ 24]. The variant sequence was of high quality with an average of $108.54 \pm 20.93$ reads per sample, mapping quality $>70$, and Phred quality scores $>35$. Furthermore, the variant was confirmed in each sample by Sanger sequencing in the forward direction using primer TGAGGTTGGGAGCCGGTAGAGG and the reverse direction using primer GTACCGACAGGGTATGTGAAGGCG (see Additional file 3: Figure S1). The segregation results were perfect, the variant was present in all the affected and was not present in any of the unaffected. We genotyped two additional unaffected members of the family, and they also lacked the variant. We searched using blat a 90 bp region containing the mutation against the human reference hg 19 and found that the region was $100 \%$ unique in the human genome. We also found that the same region was totally free of any repeats and evolutionarily conserved in vertebrates according to the comparative genomics track from the UCSC genome browser [ 25]. In summary, the ultra-rare damaging variant was present in five affected members and not present in six unaffected family members (Fig. 3a). From DNA of the family members sampled, there appears to be $100 \%$ penetrance with an autosomal dominant pattern of inheritance.

Fig. 3 

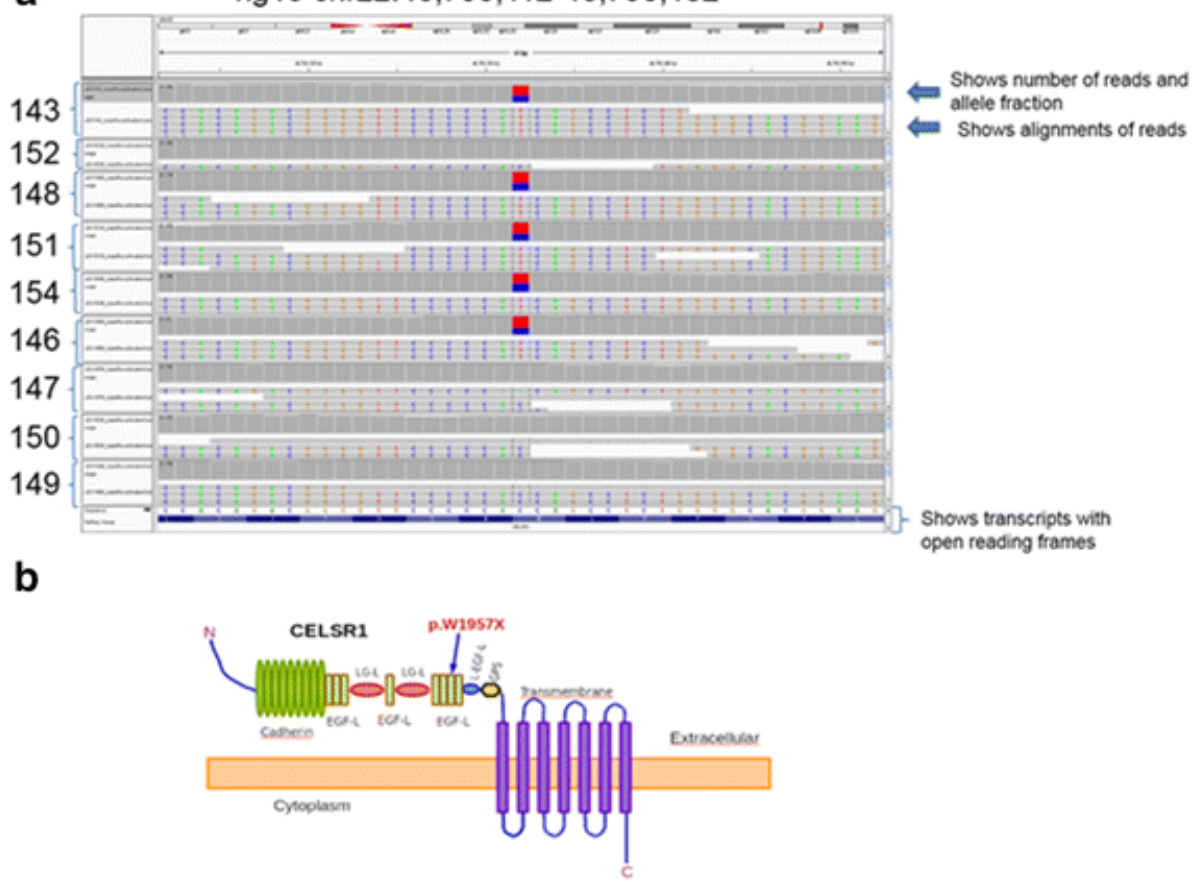

aImage from the Integrative genomics viewer, showing a 40 bp genomic region containing the variant. Each one of the samples is loaded into the panel. Each panel shows two windows, the top window displays the number of reads, and the allele fraction and the lower window show the alignments of the experimental reads. The bottom panel displays the transcripts with the open reading frames. bA cartoon representing the predicted protein structure of CELSR1 (uniprot id Q9NYQ6). An arrow represents the location of the mutation. The cartoon was inspired by the publication of Lei, et al. , 2014 [ 30]

\section{Discussion}

CELSR1 is a member of the cadherin superfamily, conserved during evolution with orthologs in invertebrate and vertebrates [26]. In addition, CELSR1 is an atypical cadherin involved in planar cell polarity. This protein has nine cadherin repeats, eight EGF-like domains, and seven transmembrane segments (Uniprot_id Q9NYQ6). CELSR1 is located at the plasma membrane with the cadherin domains acting as homophilic binding regions and the EGF-like domains involved in cell adhesion and receptor-ligand interactions. We postulate that when the mutated allele is expressed, the protein lacks the entire cadherin domain, the five EGF domains, and the LamG domains (Fig. 3b).

CELRS1 has an ortholog in Drosophila, the flamingo (Fmi). Fmi is localized at cell-cell boundaries in the wing. In the absence of Fmi, planar polarity was distorted [27]. In humans and rodents, there are three genes CELSR1-3 and Celsr13. Celsr1 and Celsr2 expression is observed during gastrulation and within the developing nervous system. Celsr3 transcripts are found only at sites of active neurogenesis [28] and mutations in CELSRI are associated with neural tube defects in humans $[29,30]$.

Recent biological evidence reported in the literature supports the causal role of CELSRI for LE in the family pedigree. Tatin et al. recently showed that CELSR1 and VANGL2 play critical roles in the complex morphogenetic process of intraluminal valve formation in murine lymphatic vessels. In their work, they demonstrated that during valve leaflet formation, endothelial cells recruit CELSR1 and VANGL2 from filopodia to discrete membrane domains at cell-cell contacts. Furthermore, mouse mutants Crsh (Celsr1) and Looptail (Vang12) show valve aplasia [ 31].

The proband displayed a common lymphatic phenotype consisting of extensive dermal backflow, tortuous vessels, as well as unique "sheet-like flow" in both legs indicating a defect in the valvular mechanism of lymph propulsion in collecting vessels. While we have not conducted functional studies of the CELSRI mutation identified in this family, the phenotype is consistent with the phenotype described in the mouse mutant Crsh [31].

In this family, the phenotype was not apparent in any living males, but obviously was passed to daughters through male parents, both now deceased and not available for clinical diagnosis or DNA collection. Three living males in the third and fourth generations were not reported to have lymphedema, but unfortunately not available for clinical diagnosis and genotyping. As a result, we are unable to determine whether penetrance is limited to females.

\section{Conclusion}

This report provides the first evidence that defective planar cell polarity signaling pathway may participate as the cause of primary, non-syndromic lymphedema in humans. 


\section{Consent}

Written informed consent for images contained in this report was obtained from the patient's parents in accordance with the guidelines of the University of Texas Health Science Center Institutional Review Board.

\section{Abbreviations}

- ICG: indocyanine green

- NIRFLI: near-infrared fluorescence lymphatic imaging

- LE: lymphedema

\section{Declarations}

\section{Additional files}

Additional file 1: Video 1.

The abnormal NIRFLI movie of lymphatic function in the left foot of the proband showing unusual lymph flow from the dorsal foot injections. (AVI $4088 \mathrm{~kb}$ )

Additional file 2: Video 2.

The normal NIRFLI movie of lymphatic function in the left foot of the asymptomatic mother showing typical lymph flow from the dorsal foot injections within collecting lymphatic vessels. (AVI $5480 \mathrm{~kb}$ ) Additional file 3: Figure S1.

A composite picture of two electropherograms comparing the Sanger sequencing results from two members of a family with lymphedema. The top panel represents the WT sequence of an unaffected family member (\#152). The low panel represents the heterozygous mutation in the affected index patient (\#143). The validation was performed as described previously [32] using the following primers: forward TGAGGTTGGGAGCCGGTAGAGG and reverse GTACCGACAGGGTATGTGAAGGCG. (TIF $201 \mathrm{~kb})$

\section{Competing interests}

JCR and EMS are listed as inventors on patents related to near-infrared fluorescence lymphatic imaging. JCR has received fees for consulting from NIRF Imaging, Inc., a UTHSCH start-up company seeking to commercialize NIRFLI technology. JCR and EMS may receive future financial benefit from NIRF Imaging, Inc. The other authors declare no potential conflict of interest.

\section{Authors' contributions}

All authors have either written parts of, reviewed, or offered changes to the manuscript. Bioinformatics $(M G-G)$, manuscript writing (MG-G, JCR, EMS-M); interpretation of results (MG-G, PK, EMS-M, PEL), imaging (MBA, $J C R)$, clinical examination and diagnoses $(R G)$, study design $(M G-G, E M S-M)$. All read and approved the final manuscript.

\section{References}

1. Mortimer PS Rockson SG New developments in clinical aspects of lymphatic disease J Clin Invest $20141243915921393826110.1172 / J C I 71608$

2. Ferrell RE, Levinson KL, Esman JH, Kimak MA, Lawrence EC, Barmada MM, Finegold DN. Hereditary lymphedema: evidence for linkage and genetic heterogeneity. Hum Mol Genet. 1998;7:2073-2078. View Article Google Scholar

3. Brouillard P Boon L Vikkula M Genetics of lymphatic anomalies J Clin Invest 20141243898 9043938256 10.1172/JCI71614

4. Mendola A Schlogel MJ Ghalamkarpour A Irrthum A Nguyen HL Fastre E Bygum A Vleuten C Fagerberg $C$ Baselga E Mutations in the VEGFR3 signaling pathway explain $36 \%$ of familial lymphedema Mol Syndromol 2013462572663776465 10.1159/000354097

5. Sevick-Muraca EM. Translation of near-infrared fluorescence imaging technologies: emerging clinical applications. Annu Rev Med. 2012;63:217-231.

View Article Google Scholar 
6. Rasmussen JC Tan IC Marshall MV Adams KE Kwon S Fife CE Maus EA Smith LA Covington KR Sevick-Muraca EM Human Lymphatic Architecture and Dynamic Transport Imaged Using Nearinfrared Fluorescence Transl Oncol 2010363623723000461 10.1593/tlo.10190

7. Rasmussen JC Tan IC Marshall MV Fife CE Sevick-Muraca EM Lymphatic imaging in humans with near-infrared fluorescence Curr Opin Biotechnol 200920174822692490 10.1016/j.copbio.2009.01.009

8. McKenna A Hanna M Banks E Sivachenko A Cibulskis K Kernytsky A Garimella K Altshuler D Gabriel S Daly M The Genome Analysis Toolkit: a MapReduce framework for analyzing nextgeneration DNA sequencing data Genome Res 2010209129713032928508 10.1101/gr.107524.110

9. Cingolani P, Platts A, Wang L, Coon M, Nguyen T, Wang L, Land SJ, Lu X, Ruden DM. A program for annotating and predicting the effects of single nucleotide polymorphisms, SnpEff: SNPs in the genome of Drosophila melanogaster strain w1118; iso-2; iso-3. Fly (Austin). 2012;6:80-92.

View Article Google Scholar

10. Yang H, Wang K. Genomic variant annotation and prioritization with ANNOVAR and wANNOVAR. Nat Protoc. 2015;10:1556-1566.

View Article Google Scholar

11. Adzhubei I, Jordan DM, Sunyaev SR. Predicting functional effect of human missense mutations using PolyPhen-2. Curr Protoc Hum Genet. 2013;Chapter 7:Unit7 20.

12. Ng PC Henikoff S SIFT: Predicting amino acid changes that affect protein function Nucleic Acids Res $200331133812381416891610.1093 /$ nar/gkg509

13. Schwarz JM, Rodelsperger C, Schuelke M, Seelow D. MutationTaster evaluates disease-causing potential of sequence alterations. Nat Methods. 2010;7:575-576.

View Article Google Scholar

14. Liu X, Wu C, Li C, Boerwinkle E. dbNSFP v3.0: A One-Stop Database of Functional Predictions and Annotations for Human Non-synonymous and Splice Site SNVs. Hum Mutat. 2015. doi: 10.1002/humu.22932

15. Tennessen JA Bigham AW O'Connor TD Fu W Kenny EE Gravel S McGee S Do R Liu X Jun G Evolution and functional impact of rare coding variation from deep sequencing of human exomes Science 2012337609064693708544 10.1126/science.1219240

16. Abecasis GR, Altshuler D, Auton A, Brooks LD, Durbin RM, Gibbs RA, Hurles ME, McVean GA. A map of human genome variation from population-scale sequencing. Nature. 2010;467:1061-1073.

View Article Google Scholar

17. Robinson JT Thorvaldsdottir H Winckler W Guttman M Lander ES Getz G Mesirov JP Integrative genomics viewer Nat Biotechnol 201129124263346182 10.1038/nbt.1754

18. The Cancer Genome Atlas [ http://cancergenome.nih.gov/].

19. The Scripps Wellderly Study [ ftp://stsi-ftp.sdsc.edu/pub/wellderly/].

20. Exome Aggregation Consortium (ExAC) [ http://exac.broadinstitute.org].

21. Landrum MJ Lee JM Benson M Brown G Chao C Chitipiralla S Gu B Hart J Hoffman D Hoover J ClinVar: public archive of interpretations of clinically relevant variants Nucleic Acids Res 201644 D1 D862 8684702865 10.1093/nar/gkv1222

22. Amberger JS Bocchini CA Schiettecatte F Scott AF Hamosh A OMIM.org: Online Mendelian Inheritance in Man (OMIM(R)), an online catalog of human genes and genetic disorders Nucleic Acids Res 201543 Database issue D789 7984383985 10.1093/nar/gku1205

23. Forbes SA Beare D Gunasekaran P Leung K Bindal N Boutselakis H Ding M Bamford S Cole C Ward S COSMIC: exploring the world's knowledge of somatic mutations in human cancer Nucleic Acids Res 201543 Database issue D805 8114383913 10.1093/nar/gku1075 
24. Hakenberg $J$ Cheng WY Thomas $P$ Wang YC Uzilov AV Chen R Integrating 400 million variants from 80,000 human samples with extensive annotations: towards a knowledge base to analyze disease cohorts BMC Bioinformatics 2016171244706706 10.1186/s12859-015-0865-9

25. Rosenbloom KR Armstrong J Barber GP Casper J Clawson H Diekhans M Dreszer TR Fujita PA Guruvadoo L Haeussler M The UCSC Genome Browser database: 2015 update Nucleic Acids Res 201543 Database issue D670 6814383971 10.1093/nar/gku1177

26. Halbleib JM, Nelson WJ. Cadherins in development: cell adhesion, sorting, and tissue morphogenesis. Genes Dev. 2006;20:3199-3214.

View Article Google Scholar

27. Kimura H, Usui T, Tsubouchi A, Uemura T. Potential dual molecular interaction of the Drosophila 7pass transmembrane cadherin Flamingo in dendritic morphogenesis. J Cell Sci. 2006;119:1118-1129. View Article Google Scholar

28. Formstone CJ, Little PF. The flamingo-related mouse Celsr family (Celsr 1-3) genes exhibit distinct patterns of expression during embryonic development. Mech Dev. 2001;109:91-94.

View Article Google Scholar

29. Allache R, Marco P, Merello E, Capra V, Kibar Z. Role of the planar cell polarity gene CELSRI in neural tube defects and caudal agenesis. Birth Defects Res A Clin Mol Teratol. 2012;94:176-181.

View Article Google Scholar

30. Lei Y Zhu H Yang W Ross ME Shaw GM Finnell RH Identification of novel CELSRI mutations in spina bifida PLoS One 2014933954890 10.1371/journal.pone.0092207

31. Tatin F Taddei A Weston A Fuchs E Devenport D Tissir F Makinen T Planar cell polarity protein Celsr 1 regulates endothelial adherens junctions and directed cell rearrangements during valve morphogenesis Dev Cell 201326131443714594 10.1016/j.devcel.2013.05.015

32. Agollah GD Gonzalez-Garay ML Rasmussen JC Tan IC Aldrich MB Darne C Fife CE Guilliod R Maus EA King PD Evidence for SH2 domain-containing 5'-inositol phosphatase-2 (SHIP2) contributing to a lymphatic dysfunction PLoS One 20149114226566 10.1371/journal.pone.0112548 